\title{
Conformation of Tamarind Seed Xyloglucan Oligomers
}

\author{
Shigenobu Yamanaka*1, Mituru Mimura*1, Hiroshi Urakawa*1, Kanji Kajiwara*1, \\ Mayumi Shirakawa ${ }^{* 2}$ and Kazuhiko Yamatoya ${ }^{* 2}$
}

\author{
${ }^{* 1}$ Faculty of Engineering and Design, Kyoto Institute of Technology, Kyoto, Sakyo-ku, Matsugasaki, \\ 606-8585 Japan \\ *2Dainippon Pharmaceutical Co., Ltd., Osaka, Suita, Enoki-cho 33-94, 564-0053 Japan
}

\begin{abstract}
Tamarind seed polysaccharide (TSP) xyloglucan consists of a $(1 \rightarrow 4)$ - $\beta$-D-glucan spine (cellulose spine) with $(1 \rightarrow 6)-\alpha$-branched xylose, which is partially substituted by $(1 \rightarrow 2)-\beta$-galacto-xylose. Thus TSP xyloglucan yields three types of monomer units (heptasaccharide DP7, octasaccharide DP8 and nonasaccharide $D P 9$ where $D P$ denotes the number of residues), which differs in the number of galactose side chains. Three types of monomers were prepared and their conformation in water was elucidated in detail by means of small-angle X-ray scattering and molecular dynamics. Dimers, trimers and tetramers (constituted of a mixture of arbitrary combinations of three types of monomers) were also prepared and their conformation was analyzed from the results of small-angle X-ray scattering. The results confirm the thin ribbon-like structure of xyloglucan oligomers. The conformation of xylose and galacto-xylose branches was also discussed.
\end{abstract}

\section{Introduction}

TSP xyloglucan (TSP; tamarind seed polysaccharide) used as a thickener in the food processing industry, is extracted from the seeds of Tamarindus indica. The primary structure of TSP xyloglucan consists of a $(1 \rightarrow 4)-\beta$-D-glucan spine (cellulose spine) with $(1 \rightarrow 6)-\alpha$-branched xylose, which is partially substituted by $(1 \rightarrow 2)-\beta$-galacto-xylose. Thus the enzymatic degradation of the backbone chain yields three types of monomer units (heptasaccharide $D P 7$, octasaccharide $D P 8$ and nonasaccharide $D P 9$ where $D P$ denotes the number of residues) as shown in Fig. 1, which differs in the number of galactose side chains [1]. Oligomers with less galactose side chains tend to aggregate because the loss of galactose side chains reduces hydrophilicity. In fact the enzymatic degradation by $\beta$-galactosidase results in the gelation of TSP xyloglucan aqueous solution at room temperature when about $50 \%$ of galactose residues are released[2].
The conformation of an isolated native TSP xyloglucan is less understood, although it is conjectured to assume an extended zigzag chain conformation from the analogy of cellulose[3]. Native TSP xyloglucan shows a strong tendency of lateral aggregation in aqueous solution and the chain stiffness depends on the number of aggregated strands[4]. An attempt is made to elucidate the intrinsic properties of TSP xyloglucan by chemical modification as this practice has been proved to be effective in the case of cellulose. The chemical modification (the enzymatic oxidation) of native TSP xyloglucan introduces electrical charges onto TSP xyloglucan and is expected to improve its solubility in water. The carboxylated TSP xyloglucan revealed high chain stiffness (the Kuhn length $l_{k} \geq 140$ $\mathrm{nm}$ ), but 3 to 5 chains are still laterally aggregated as the evaluated values of the mass per unit length $M_{L}$ indicated[5].

In this paper, we examine the conformation of TSP xyloglucan oligomers by means of the small-angle $\mathrm{X}$-ray scattering and the molecular dynamics in order 
to analyze quantitatively the conformation of an isolated TSP xyloglucan chain. Since TSP xyloglucan monomer units are classified into three types according to the side chains (Fig. 1), the effect of side chains on the conformation will be characterized by preparing three types of monomers and mixed dimers/trimers containing various conformers. Here the molecular dynamics is applied on each monomer in the presence of water, and the particle scattering factor is calculated as an ensemble average of generated chains from the atomic coordinates according to the Debye formula. The resulted scattering profiles are compared with SAXS profiles observed from the aqueous solutions of corresponding oligomers. A simulated chain will thus visualize the conformation of TSP xyloglucan oligomers.

DP7

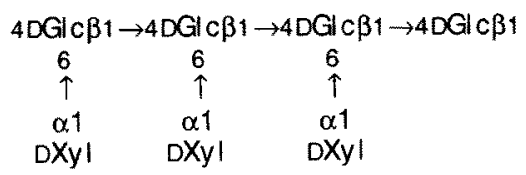

DP8 (1) $4 \mathrm{DGl} c \beta 1 \rightarrow 4 \mathrm{DGl} \mathrm{c \beta 1} \rightarrow 4 \mathrm{DGl} c \beta 1 \rightarrow 4 \mathrm{DGl} c \beta 1$

$\begin{array}{ccc}6 & 6 & 6 \\ \uparrow & \uparrow & \uparrow \\ \alpha 1 & \alpha 1 & \alpha 1 \\ \text { DXyl } & \text { DXyl } & \text { DXyl } \\ & & 2 \\ & & \uparrow \\ & & \beta 1 \\ & & \text { DGal }\end{array}$

DP8 (2) 4 DGl c $\beta 1 \rightarrow 4 \mathrm{DGl} C \beta 1 \rightarrow 4 \mathrm{DGl} C \beta 1 \rightarrow 4 \mathrm{DGl} \mathrm{c} \beta 1$

$\begin{array}{ccc}6 & 6 & 6 \\ \uparrow & \uparrow & \uparrow \\ \alpha 1 & \alpha 1 & \alpha 1 \\ \text { DXyl } & \text { DXyl } & \text { DXyl } \\ & 2 & \\ & \uparrow & \\ & \beta 1 & \\ & \text { DGal } & \end{array}$

DP9 $\quad 4 D G l c \beta 1 \rightarrow 4 D G 1 c \beta 1 \rightarrow 4 D G l c \beta 1 \rightarrow 4 D G \mid c \beta 1$

$\begin{array}{ccc}6 & 6 & 6 \\ \uparrow & \uparrow & \uparrow \\ \alpha 1 & \alpha \uparrow & \alpha 1 \\ \text { DXyl } & \text { DXyl } & \text { DXyl } \\ & 2 & 2 \\ & \uparrow & \uparrow \\ & \beta 1 & \beta 1 \\ & \text { DGal } & \text { DGal }\end{array}$

Fig. 1 Schematic representation of TSP xyloglucan monomers

\section{Experimental}

\subsection{Materials}

\subsubsection{Preparation of xyloglucan monomers}

TSP xyloglucan monomers (heptasaccharide, octasaccharide and nonasaccharide) were prepared from tamarind seed xyloglucan (commercially available as Glyloid from Dainippon Pharmaceutical Co. Ltd., Japan), according to the modification procedure proposed by York et al.[1]. TSP xyloglucan was purified by precipitation from its aqueous solution into 2-propanol and dried with acetone in air prior to enzymatic hydrolysis. Purified xyloglucan (lg) was dissolved in $100 \mathrm{ml}$ of $20 \mathrm{mM}$ sodium acetate buffer $(\mathrm{pH} 4.0)$ and incubated at $37^{\circ} \mathrm{C}$ for 65 hours with $16 \mathrm{mg}$ end-1,4- $\beta$-glucanase from Trichoderma vinide (Cellulase Onozuka 3S, Yakult Pharmaceutical Ind. Co. $\mathrm{Ltd}$.). The reaction mixture was heated at $95^{\circ} \mathrm{C}$ for 20 $\min$ to inactivate the enzyme and centrifuged at 8000 $\mathrm{rpm}$ for $15 \mathrm{~min}$ to remove insoluble materials. The supernatant was passed through an ion-exchange resin (SK1B, SA10A, Mitsubishi Chemical Co.), concentrated to a small volume with a rotary evaporator, and then freeze-dried. The mixture of oligosaccharides was separated by HPLC [6] as follows: The mixture of three types of monomers $(0.6 \mathrm{~g})$ was dissolved in $6 \mathrm{ml}$ of distilled water and applied at room temperature to HPLC coupled with a preparation column YMC Pack Polyamine II (20mmI.D. $\times 250 \mathrm{~mm}$, YMC Co. Ltd., Japan) and an RI detector. The flow rate was $8.0 \mathrm{ml} / \mathrm{min}$ with $50 \%$ acetonitorile as the eluent. Each monomer fraction was collected and rechromatographed on the same column.

\subsubsection{Preparation of xyloglucan dimer and higher oligomers}

Xyloglucan dimer which is constituted of an arbitrary combination of two monomers (DP of 14 to 18) was prepared from partial hydrolysis of xyloglucan. $10 \%$ xyloglucan aqueous solution was adjusted to $\mathrm{pH} 3.7$ by citric acid and incubated at $37^{\circ} \mathrm{C}$ for 64 hours with $0.4 \%$ end-1,4- $\beta$-glucanase. The reaction mixture was heated at $95^{\circ} \mathrm{C}$ for $20 \mathrm{~min}$ to inactivate the enzyme and centrifuged at $8000 \mathrm{rpm}$ for $15 \mathrm{~min}$ to remove insoluble materials. The supernatant was passed through an ion-exchange resin (SK1B, SA10A). The solution was precipitated by $60 \%$ 
2-propanol and centrifuged at $3000 \mathrm{rpm}$ for $5 \mathrm{~min}$ to collect the precipitate. The precipitate was washed by $60 \%$ 2-propanol and dried with acetone in air. The product was dissolved in distilled water (10\%), precipitated by $70 \%$ methanol and then centrifuged at $3000 \mathrm{rpm}$ for $5 \mathrm{~min}$ to remove insoluble materials. The supernatant was precipitated by $90 \%$ methanol and then centrifuged at $3000 \mathrm{rpm}$ for $5 \mathrm{~min}$ to collect the precipitate. The precipitate was washed by $90 \%$ methanol and dried with acetone in air. The content of xyloglucan dimer was analyzed at $60^{\circ} \mathrm{C}$ by $\mathrm{HPLC}$ coupled with columns Shodex SUGAR KS805 and KS $802(8 \mathrm{mmI}$.D. $\times 500 \mathrm{~mm}$, Showa Denko, Japan) and an RI detector. The flow rate was $1 \mathrm{ml} / \mathrm{min}$ with water as the eluent. The content was about $85 \%$.

TSP xyloglucan trimer and tetramer were prepared by partial hydrolysis TSP xyloglucan in a similar as dimer. Trimer is a mixture of arbitrary combinations of three monomers ( $D P$ of 21 to 27 ), and tetramer a mixture of arbitrary combinations of four monomers (DP of 28 to 36 ).

\subsection{Small-angle $\mathrm{X}$-ray scattering}

Small-angle X-ray scattering (SAXS) was observed with a SAXS equipment for solutions installed at BL-10C in the Photon Factory, Tsukuba, Japan. An incident beam from synchrotron radiation was monochromatized to $\lambda=0.140 \mathrm{~nm}$ with a double-crysta monochorometer and focused to the position of the sample cell $1 \mathrm{~cm} \times 0.5 \mathrm{~cm} \times 0.2 \mathrm{~cm}$ (made of glass with quart $z$ windows of $20 \mu \mathrm{m}$ thick) with a bent focusing mirror. The scattered $\mathrm{X}$-ray was monitored by a one-dimensional position sensitive proportional counter (the effective length $190 \mathrm{~mm}$ ) positioned at the distance of about 1 meter from the sample holder. The exact camera length was calibrated by the diffraction peaks of collagen fiber. The observed range was from $q=0.2 \mathrm{~nm}^{-1}$ to $q=4.5 \mathrm{~nm}^{-1}$ in terms of the magnitude of scattering vector $q(\equiv(4 \pi / \lambda) \sin (\theta / 2)$ with $\lambda$ and $\theta$ being the wavelength of the incident beam and the scattering angle, respectively).

The conventional analysis of the SAXS profiles yields the radius of gyration $R_{G}$ or the cross-sectional radius of gyration $R_{G c}$ from the slope of the linear part when plotted $\ln I(q)$ (the Guinier plot) or $\ln q I(q)$ (the cross-sectional Guinier plot) against $q^{2}$, respectively.

\subsection{Molecular dynamics}

Computer generated three types of monomer units (heptasaccharide, octasaccharide and nonasac charide), and the molecular mechanics minimizatior was applied to each generated monomer chain. The resulted model by the molecular mechanics minimization was annealed by affording a thermal disturbance, and then was energy-minimized in the second time by the molecular mechanics. Here each generated monomer was placed together with 500 water molecules in the cell of $2.557 \times 2.557 \times 2.557 \mathrm{~nm}^{3}$ Then the temperature was fixed to $25^{\circ} \mathrm{C}$, and the molecular dynamics was performed with the CFF91 force field. The computation was made for $100 \mathrm{ps}$ time history.

The particle scattering factor was calculated from the atomic coordinates of each simulated xyloglucan oligomer according to the Debye formula, that is, the scattering profile is given by

$$
I(q) \approx \sum_{i=1}^{n} g_{i}^{2} \phi_{i}^{2}(q)+2 \sum_{i=1}^{n-1} \sum_{j=i+1}^{n} g_{i} g_{j} \phi_{i}(q) \phi_{j}(q) \cdot \frac{\sin d_{i j q}}{d_{i j} q}
$$

where $q$ denotes the magnitude of a scattering vector given by $(4 \pi / \lambda) \sin \theta$ with $\theta$ and $\lambda$ being a half of the scattering angle and the wavelength of an incident $\mathrm{X}$-ray, respectively. $g_{i}$ is an atomic scattering factor and $d_{i j}$ is the distance between the $i$ th and the $j$ th atoms. The form factor for the $i$ th atom $\phi_{i}(q)$ is assumed to be given by the form factor of a sphere having a radius equivalent to a van der Waals radius of the $i$ th atom as

$$
\phi_{i}(q)=\frac{3\left[\sin \left(R_{i} q\right)-\left(R_{i} q\right) \cos \left(R_{i} q\right)\right]}{\left(R_{i} q\right)^{3}}
$$

with $R_{i}$ being the van der Waals radius of the $i$ th atom.

\section{Results and Discussion}

\subsection{TSP xyloglucan monomer}

The small-angle X-ray scattering (SAXS) was observed from the aqueous solutions of heptasaccharide, octasaccharide and nonasaccharide of TSP xyloglucan monomer units at room temperature as shown in Fig. 2. The observed SAXS indicates the presence of aggregates in the aqueous solutions of heptasaccharide and octasaccharide as confirmed by the upturn of the scattered intensity at $q \rightarrow 0$ in the double logarithmic plots (not shown), while no such aggregation was observed in the aqueous solution of nonasaccharide. The lack of galactosic units 

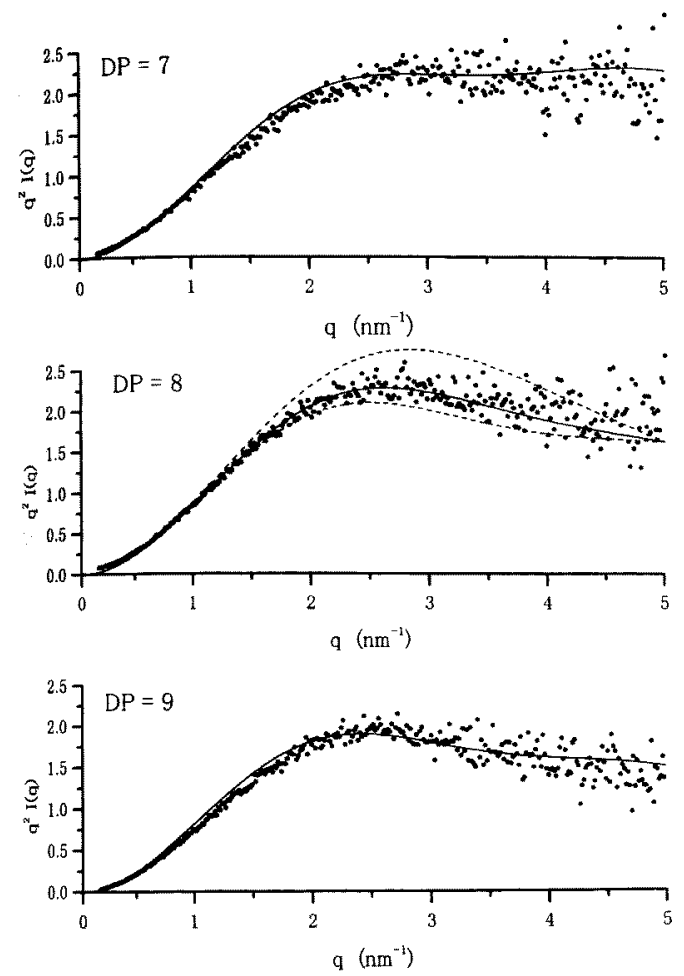

Fig. 2 Small-angle X-ray scattering observed (black circles) and calculated (solid or dotted lines) from three types of TSP xyloglucan monomer units (heptasaccharide, octasaccharide and nonasaccharide). Octasaccharide has two isomers, and the solid line represents the calculated profile as the average of those from the two isomers ( $30 \%$ octasaccharide 1 and $70 \%$ octasaccharide 2 ). The dotted lines correspond to the scattering profiles from the octasaccharide 1 (above) and the octasaccharide 2 (below). See Figs. 1 and 3 for the specification of the octasaccharides 1 and 2 .

seems to promote aggregation.

The observed SAXS profiles were analyzed conventionally to evaluate the radius of gyration $R_{C}$ and the cross-sectional radius of gyration $R_{C c}$ from the respective Guinier plots as summarized in Table 1 . The effect of aggregation appears at lower $q$ range, so that the corresponding radius of gyration was evaluated from the linear portion in the respective Guinier plots satisfying the condition that $q R_{C} \cong 1$ or $q R_{G r} \cong 1$. In order to avoid the ambiguity due to the narrow Guinier range
Table 1 Radius of gyration $R_{G}$, the cross-sectional radius of gyration $R_{G c}$ and 3 semi-axes $a, b$ and $c$ of the model ellipsoid representing the shape of TSP xyloglucan heptasaccharide, octasaccharide and nonasaccharide

\begin{tabular}{|c|c|c|c|}
\hline TSP xyloglucan & $R_{G} / \mathrm{nm}$ & $R_{G d} / \mathrm{nm}$ & $a \times b \times c / \mathrm{nm}^{3}$ \\
\hline $\begin{array}{c}\text { TSP monomer } \\
\text { Heptasaccharide }\end{array}$ & $0.73^{\mathrm{a}}$ & $0.32^{\mathrm{b}}$ & $0.22^{\mathrm{d}} \times 0.62^{\mathrm{d}} \times 1.49^{\mathrm{d}}$ \\
$\begin{array}{c}\text { Octasaccharide } \\
\text { Nonasaccharide }\end{array}$ & $0.72^{\mathrm{a}}$ & $0.37^{\mathrm{b}}$ & $0.22^{\mathrm{d}} \times 0.71^{\mathrm{d}} \times 1.43^{\mathrm{d}}$ \\
\hline $\begin{array}{c}\text { TSP dimer } \\
D P=14 \sim 18\end{array}$ & $1.12^{\mathrm{a}}$ & $0.36^{\mathrm{b}}$ & $0.23^{\mathrm{d}} \times 0.75^{\mathrm{d}} \times 1.41^{\mathrm{d}}$ \\
\hline $\begin{array}{c}\text { TSP trimer } \\
D P=21 \sim 27\end{array}$ & $1.62^{\mathrm{a}}$ & $0.39^{\mathrm{b}} \times 2.40^{\mathrm{d}}$ \\
\hline $\begin{array}{c}\text { TSP tetramer } \\
D P=28 \sim 36\end{array}$ & - & $0.22^{\mathrm{e}} \times 0.75^{\mathrm{d}} \times 3.54^{\mathrm{d}}$ \\
\hline $\begin{array}{c}(1 \rightarrow 4) \text { - } \beta \text {-D-glucan } \\
\text { spine }\end{array}$ & - & 0.21 & \\
\hline
\end{tabular}

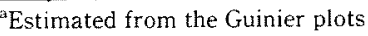

${ }^{\circ}$ Estimated from the Guinier plots for cross-section

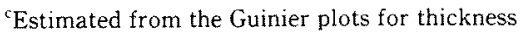

${ }^{a}$ Calculated by assuming an ellipsoidal shape

eAssumed

for monomers, the tri-axial body fitting was examined for the observed SAXS profiles from TSP xyloglucan monomers. Each profile was found to be represented by an ellipsoid[7], whose dimensions are listed in Table 1. The ellipsoid dimensions are consistent with the radius of gyration and the cross-sectional radius of gyration evaluated from the observed SAXS profiles, where $R_{G}{ }^{2}$ and $R_{G c}{ }^{2}$ are calculated as $\left(a^{2}+b^{2}+c^{2}\right) / 5$ and $\left(b^{2}+c^{2}\right) /$ 4 from the model ellipsoids. The radius of gyration seems almost invariant with the molecular weight, although the cross-sectional radius of gyration (caluclated as was found to increase slightly with the number of residues in side chains. The removal of galactose side chains causes thinning of the cross-section of TSP xyloglucan. The cross-sectional radius of gyration of xyloglucan monomers is considerably larger than that evaluated for $(1 \rightarrow 4)-\beta$-Dglucan spine $(0.21 \mathrm{~nm}$ for cellulose) [8], indicating the xylose or galacto-xylose branches protruding from the spine. Another characteristics of the xyloglucan cross-section is its flatness. In the elliptic models fitted to each monomer, the length of the shortest semi-axis 
is constant as $0.22 \mathrm{~nm}$ for all three types of monomers. The width of the cross-section increases from $0.62 \mathrm{~nm}$ (heptasaccharide) to $0.71 \mathrm{~nm}$ (octasaccharide) and 0.75 nm (nonasaccharide) as the number of residues in side chains increases. The results indicate the side chains protruding horizontally from the flat $(1 \rightarrow 4)-\beta$-D-glucan spine surface. The longest axis may represent the spine length, which should be the same among three types of monomers if the spine conformation is identical. However, a shorter length was found for a larger monomer, suggesting a more arched structure.

Three types of TSP xyloglucan monomers were
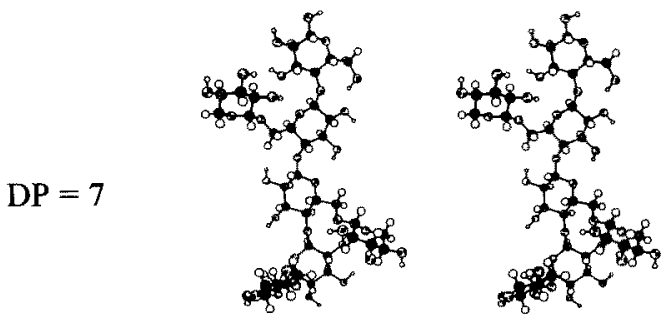

$\mathrm{DP}=8$

(1)
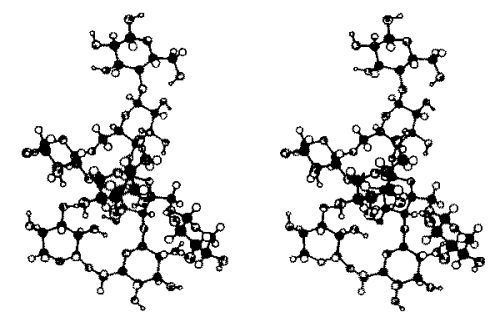

$\mathrm{DP}=8$
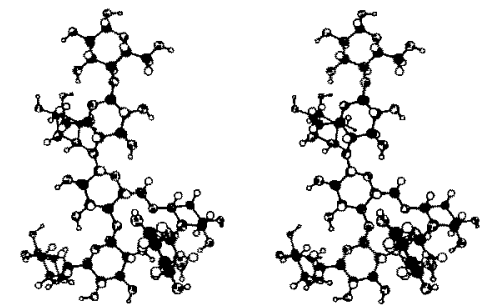

$\mathrm{DP}=9$
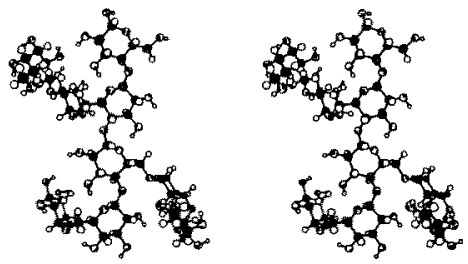

Fig. 3 Simulated TSP xyloglucan monomer units (stereo figure) simulated by molecular dynamics in order to elucidat the details of chain conformation. The particlk scattering factors were calculated according to eq. (1) from the atomic coordinates of the simulatec xyloglucan monomer units schematically shown ir Fig. 1. The results are shown in Fig. 2, where the solic lines show the calculated scattering profiles. The agreement with the observed SAXS profiles is satisfactory, so that the simulated chains represent the conformation of respective TSP xyloglucan monomer units in water. The simulated monomer units are showr in Fig. 3, where respective $(1 \rightarrow 4)$ - $\beta$-D-glucan spines art

$\mathrm{DP}=7$
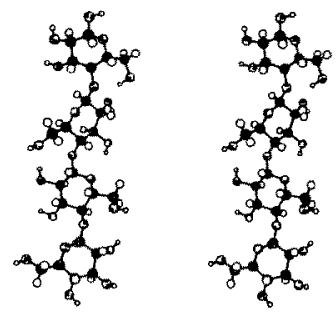

$\mathrm{DP}=8$

(1)
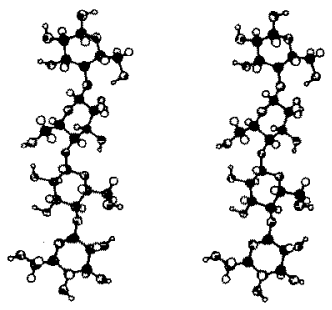

$\mathrm{DP}=8(2)$
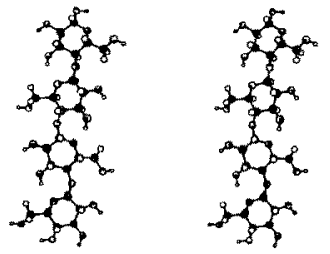

$\mathrm{DP}=9$
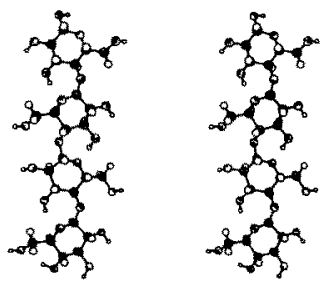

Fig. 4 Simulated $(1 \rightarrow 4)-\beta$-D-glucan spines of each TSP xyloglucan monomer (stereo figure) 
also shown. Fig. 4. Two isomers are available for TSP xyloglucan octasaccharide (also see Fig. 1). Both isomers were simulated and the corresponding scattering profiles were calculated. Since TSP xyloglucan octasaccharide consists of the mixtures of two isomers, the scattering profile was calculated as a weighted average $(30: 70$ in this instance, see the caption of Fig. 2) of those scattered from two isomers in order to fit the observed SAXS profile. The dotted lines show the scattering profiles corresponding to respective octasaccharide isomers.

Here $(1 \rightarrow 4) \cdot \beta$-D-glucan spines were found to assume rather flat conformation, and xylose and galacto-xylose branches to extend and fold upright on the $(1 \rightarrow 4)-\beta$-D-glucan flat surface. The conformation is similar to the results by Levy et al.[3] who simulated the conformation of TSP xyloglucan side chains by assuming flat (cellulose-like) and twisted (cellobiose-like) backbone conformation. The twisted backbone conformation is supposed to be preferred in solution[3].

In order to elucidate the effect of xylose branches on thespine conformation, the expected scattering profiles from the $(1 \rightarrow 4) \cdot \beta$-D-glucan spine (cellulose spine) were calculated from the simulated TSP xyloglucan monomer units (see Fig. 5). The scattering profiles are slightly different from each other, reflecting the difference of the chain conformation. Nonasaccharide seems to have a flat arched spine conformation, while octasaccharide and heptasaccharide assume a slightly twisted conformation. The loss of galactose side chains

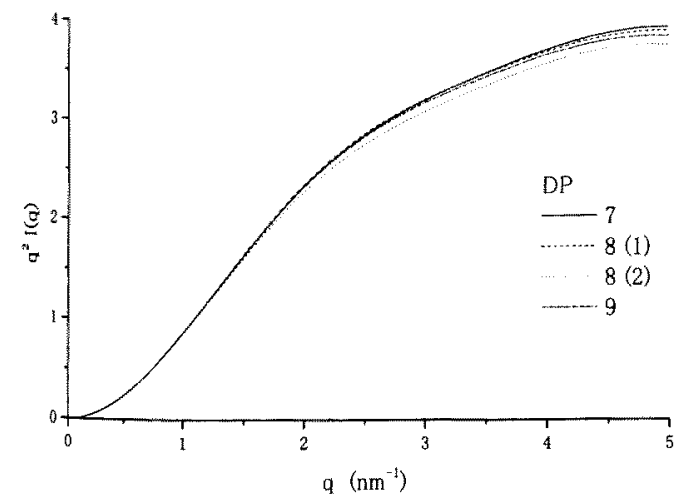

Fig. 5 Scattering profiles expected from the $(1 \rightarrow 4)$. $\beta \cdot D$-glucan spine (cellulose spine) of each TSP xyloglucan monomer unit
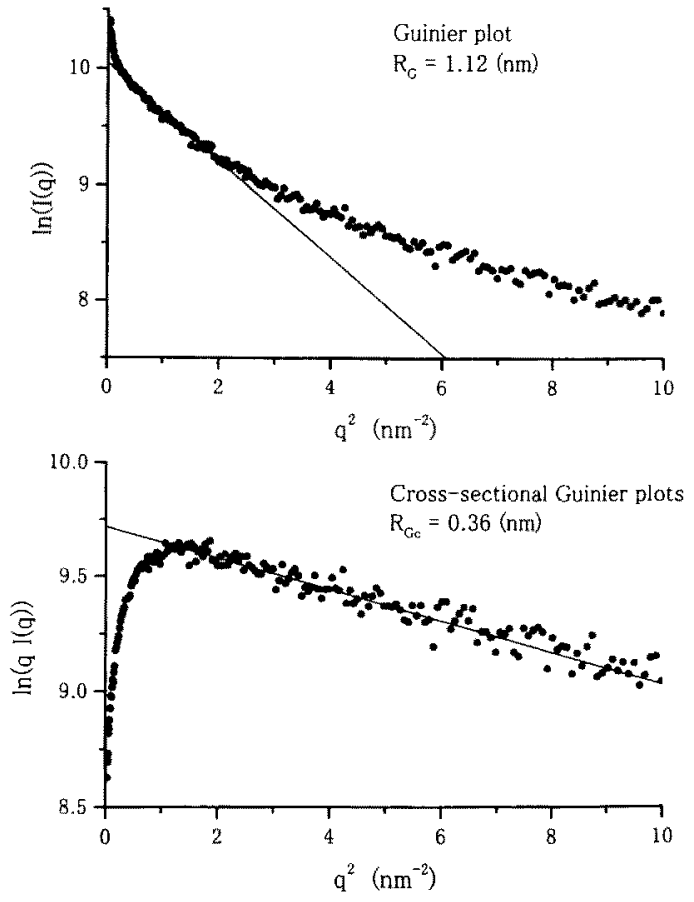

Fig. 6 SAXS profile observed from TSP xyloglucan dimer in aqueous solution (Guinier plots and cross-sectional Guinier plots)

results in the increase of hydrophobicity and seems to twist the backbone.

\subsection{TSP xyloglucan dimer/trimer/tetramer}

The SAXS profile from TSP xyloglucan dimer shows considerable aggregation as shown in Fig. 6 . The radius of gyration $R_{G}$ and the cross-sectional radius of gyration $R_{G}$ were evaluated from the conventional Guinier plots ignoring the data at lower scattering angles as summarized in Table 1. Assuming an elliptic shape, the longest semi-axis is calculated as $2.40 \mathrm{~nm}$ from the values of two radii $R_{G}$ and $R_{G c}$ estimated from the respective Guinier plots of observed SAXS intensities. The cross-sectional radius of gyration is almost identical to those estimated for the monomers, but, due to the thin arched structure, the longest semi-axis length is shorter than the double of the longest semi-axis length estimated for monomers.

The cross-sectional radii of gyration of TSP xyloglucan trimer and tetramer are estimated as 0.39 $\mathrm{nm}$ and $0.37 \mathrm{~nm}$, respectively, from the cross-sectional Guinier plots (see Table 1). These values are identical 
to those estimated for TSP xyloglucan monomers and dimer. The results confirm the stiff nature of TSP xyloglucan, which has a thin ribbon-like structure. An elliptic shape was assumed for TSP xyloglucan trimer and the length of spine was estimated as $3.54 \mathrm{~nm}$ (Table 1) using the values of $R_{G}$ and $R_{G r}$ estimated from the respective Guinier plots of observed SAXS intensities. Although the value is not exactly equal to 3 times of the monomer length, it seems that an additional monomer unit adds approximately $1 \mathrm{~nm}$ to the total length of the spine. This increase of the spine length per monomer unit is reasonable when considered the slightly arched flat $(1 \rightarrow 4)-\beta$-D-glucan spine structure.

\section{Conclusion}

The molecular dynamics combined with the small-angle $X$-ray scattering technique revealed a slightly arched $(1 \rightarrow 4)$ - $\beta$-D.glucan spin structure of TSP xyloglucan oligomer. TSP xyloglucan is constituted of three types of monomer units differing in the number of galactose side chains. Here $(1 \rightarrow 4)-\beta$-D-glucan spines were found to assume rather flat ribbon-like structure, and $x y$ lose and galacto-xylose branches to protrude and fold upright on the $(1 \rightarrow 4)-\beta$-D-glucan flat surface. That is, TSP xyloglucan oligomer is characterized as a stiff ribbon and an additional monomer unit adds approximately $1 \mathrm{~nm}$ to the total length of $(1 \rightarrow 4) \cdot \beta \cdot \mathrm{D}$ - glucan spine.

\section{Acknowledgements}

This work was performed under the approval of the Photon Factory Advisory Committee (Proposal No. 91-217).

\section{References}

1. W. S. York, H. van Halbeek, A. G. Darvill, and P. Albersheim, Carbohydr. Res., 200, 9(1990).

2. M. Shirakawa, K. Yamatoya, and K. Nishinari, Food Hydrocoll., 12, 25 (1998).

3. S. Levy, W. S. York, R. Stuike-Prill, B. Meyer, and A. L. Staehelin, Plant J., 1, 195(1991).

4. P. Lang, and K. Kajiwara, J. Biomater. Sci., 4, 517 (1993).

5. P. Lang, K. Kajiwara, and W. Burchard, Macromolecules, 26, 3992 (1993).

6. T. Hayashi, T. Takeda, K. Ogawa and Y. Mitsuishi, Plant Cell Physiol., 35, 893(1994).

7. Y. Yuguchi, M. Mimura, H. Urakawa, K. Kajiwara, M. Shirakawa, K. Yamatoya, S. Kitamura, Proc. Intern. Workshop Green Polym., p.306, Bandung-Bogor, Indonesia, 1996

8. M. Mimura, H. Urakawa, K. Kajiwara, S. Kitamura, K. Takeo, Macromol. Symp., 99, 43 (1995). 